\title{
Dynamical flavour dependence of static heavy meson decay constants on the lattice
}

\author{
G. M. de Divitiis, R. Frezzotti, M. Masetti and R. Petronzio \\ Dipartimento di Fisica, Università di Roma Tor Vergata \\ and \\ INFN, Sezione di Roma II \\ Viale della Ricerca Scientifica, 00133 Roma, Italy
}

April 30, 1996

\begin{abstract}
We study the dynamical flavour dependence of the lattice estimates of the heavy meson decay constant in the static limit. We perform the analysis by considering negative flavour numbers and by extrapolating the results to positive values. We observe a sizeable flavour dependence which increases the quenched estimates.
\end{abstract}

ROM2F-96-16 
The knowledge of leptonic decay constants of heavy mesons enters the determination of the Cabibbo-Kobayashi-Maskawa mixing angles. In particular, the value of the $B$ meson decay constant constraints the shape of the unitarity triangle and sets the size of possible $\mathrm{CP}$ violation in $B$ decays. Non perturbative estimates of this quantity can be obtained from lattice calculations which are still confronted with various sources of systematic errors. The presence in the dynamics of a heavy meson system of two very different mass scales implies that reasonable values for exploring the $B$ meson dynamics with enough resolution for the heavy quark propagation and enough volume for the heavy meson wave function are a lattice size of the order of $1.5 \mathrm{fm}$ and a lattice spacing of order of $0.02 \mathrm{fm}$. These values lead to about 75 lattice points, beyond present computer capabilities.

Estimates for the physical region of the heavy quark mass are obtained by interpolating between the results which are obtained in the charm quark mass region and those deriving from an expansion of the fermion action in the inverse of the heavy quark mass. The first term in the expansion is the so called static limit [1] and corresponds to the approximation where the heavy quark does not propagate in space. An important source of possible systematic errors of such computations is the quenched approximation: the inclusion of dynamical quark loops could alter the quenched estimates and vanify the efforts of reducing the statistical fluctuations within the quenched approximation. In this letter we make an estimate of the unquenching effects on the pseudoscalar decay constant in the static limit with the "bermion" method [2], i.e. we extrapolate from negative to positive flavour numbers the results obtained at fixed renormalized parameters.

On the lattice and in the static limit, the correlator of two local heavylight currents is given by:

$$
G(t)=\frac{1}{L^{3} T} \sum_{x, y} \delta_{\vec{x}, \vec{y}}^{3} \delta_{t_{y}, t_{x}+t}\left\langle\operatorname{Tr}\left[P(x, y) \frac{1-\gamma_{0}}{2} S_{q}^{\dagger}(x, y)\right]\right\rangle
$$

where $S_{q}$ is the light quark propagator, $P(x, y)$ is the Polyakov line from $x$ to $y$, and $L$ and $T$ are the space and time sizes respectively.

At large times there is a single state dominating the correlation and one can extract the matrix element of the heavy-light current between such a state and the vacuum: 


$$
G(t) \stackrel{t \text { large }}{\longrightarrow} Z_{L}^{2} e^{-\Delta E t}
$$

The decay constant in the static limit can be derived from $Z_{L}$ :

$$
f_{P} \sqrt{M_{P}}=\sqrt{2} Z^{R e n} Z_{L} a^{-3 / 2}
$$

where $Z^{\text {Ren }}$ is the suitable renormalization constant of the lattice current.

In order to simulate theories with negative flavours we introduce a "bermion" action [2] which is given by $S_{g}[U]+\sum_{i} S_{\phi_{i}}\left[U, \phi_{i}\right]$, where $S_{g}$ is the standard Wilson action for the gauge sector and the second term is a sum over flavour indices of

$$
S_{\phi_{i}}\left[U, \phi_{i}\right]=\sum_{x}\left|\left[Q \phi_{i}\right](x)\right|^{2}
$$

with $\phi_{i}(x)$ the bermion field of flavour $i$. For the lattice Dirac operator we follow the standard Wilson formulation:

$$
\begin{array}{r}
{\left[Q \phi_{i}\right](x)=\frac{1}{2 \kappa} \gamma_{5} \phi_{i}(x)-\frac{1}{2} \gamma_{5} \sum_{\mu=0}^{3} U_{\mu}(x)\left(1-\gamma_{\mu}\right) \phi_{i}(x+\mu)} \\
-\frac{1}{2} \gamma_{5} \sum_{\mu=0}^{3} U_{\mu}^{\dagger}(x-\mu)\left(1+\gamma_{\mu}\right) \phi_{i}(x-\mu)
\end{array}
$$

where $\kappa$ is the Wilson hopping parameter related to the bare mass.

The square of the Dirac operator is needed for the Montecarlo update of the bermion fields and implies that the two point function of these fields represents the inverse of such a square. The usual inverse Dirac operator can be obtained by remultiplying the bermion field correlations with the Dirac operator.

The correlation $G(t)$ can be estimated with bermion field averages, in a way analogous to the one followed in [3] for the quenched case. In the quenched case, the "bermion" fields are not dynamical, they are thermalized in a frozen gauge configuration and provide a Monte Carlo inversion of the Dirac operator.

According to the method presented in [3], we define two correlators, the first corresponding to eq. 1: 


$$
G(t)=\frac{1}{n_{b} L^{3} T} \sum_{i=1}^{n_{b}} \sum_{x, y} \delta_{\vec{x}, \vec{y}}^{3} \delta_{t_{y}, t_{x}+t}\left\langle\left[Q \phi_{i}\right]^{\dagger}(x) \gamma_{5} P(x, y) \frac{1+\gamma_{0}}{2} \phi_{i}(y)\right\rangle
$$

and a second one corresponding to propagators which are the inverse of the square of the Dirac operator:

$$
G^{\left(Q^{2}\right)}(t)=\frac{1}{n_{b} L^{3} T} \sum_{i=1}^{n_{b}} \sum_{x, y} \delta_{\vec{x}, \vec{y}}^{3} \delta_{t_{y}, t_{x}+t}\left\langle\phi_{i}^{\dagger}(x) P(x, y) \frac{1+\gamma_{0}}{2} \phi_{i}(y)\right\rangle
$$

where $n_{b}$ is the number of bermion flavours over which the sum is performed.

The statistical fluctuations of these operators are reduced by the "one link integral" method [4]: at fixed bermion configuration one replaces the link by its average in the surrounding bermion and gauge configuration.

As already noticed earlier [5] the correlation function constructed from the inverse of the square of the Dirac operator in eq. 7 projects more precociously on the lowest lying state. We have therefore extracted the energy shift $\Delta E$ from this correlation and then fixed its value in the fit of the canonical correlator of eq. 6. The simulations were performed on a 25 Gigaflop machine of the APE series. The update procedure was for the gauge sector a CabibboMarinari pseudo-heatbath [6] followed by three overrelaxation sweeps and for the bermions a heat bath followed by ten overrelaxation sweeps [7]. The measurements were performed every five sweeps: we have checked that the autocorrelation time, defined as $\tau=\sigma_{\text {true }}^{2} /\left(2 \sigma_{\text {naif }}^{2}\right)$, where $\sigma_{\text {true }}$ is evaluated by collecting measurements into bins and $\sigma_{n a i f}$ is evaluated by considering each measurement as an independent one, is around 0.5 for the correlations $G(t)$ and around 1 for $G^{\left(Q^{2}\right)}(t)$. According to the procedure of extrapolating at fixed renormalized quantities, the values of the $\beta$ and $\kappa$ in each simulation with a given number of bermions were chosen to match the $\rho$ and $\pi$ masses at the corresponding quenched values. We have explored two values of the ratio $R_{2}=m_{\pi}^{2} / m_{\rho}^{2}, 0.5$ and 0.6 , with a lattice spacing corresponding to the value $\beta=5.7$ of the quenched case and appropriate values of $\kappa$.

We have made simulations with $n_{f}=-2,-4$ and -6 for $R_{2}=0.5$ and $n_{f}=-2,-4,-6$ and -8 for $R_{2}=0.6$. The quenched values are those of ref. [3] with a slight different statistics in the case of $\kappa=0.163$. The errors are evaluated as follows: we form clusters of 200 measurements. On these clusters 
we apply a jackknife algorithm to estimate the errors of the correlations. Each "jackknife cluster", defined by single elimination of a cluster from the total sample is fitted with the MINUIT minimization program. From the spread of the fits on each jackknife cluster we extract the errors that we quote for our results. For the operator of eq. 7 we have checked that the results of one and two mass fits are generally compatible while for the operator of eq. 6 a two mass fit is necessary with the lowest mass fixed by the fit of the other operator. We quote separately the statistical error of the fit and the error deriving from the uncertainty by which the correlation $G^{\left(Q^{2}\right)}(t)$ fixes the value of the lowest energy shift. All the results have been obtained on a $16^{3} \times 32$ lattice and the correlations have been taken in all directions, with a caveat. The correlations in the space directions, for values of the separation close to the boundary (beyond 11-12) may suffer from finite size effects due to the presence of dynamical "bermions". Indeed, the light quark can connect a point in the original lattice with a point sitting in the backward copy, while the heavy quark is explicitely propagated only in the forward direction and sits always in the original lattice. The result is a path wrapping around the lattice. In the quenched approximation and in the confined phase, these paths average to zero, but they are in general non zero in the unquenched case. The fermion determinant may create a fermion loop wrapping around the lattice which, through its correlation with the one from the heavy-light propagator can give to the latter a non zero expectation value. The effect is visible beyond the statistical errors for $t>10$. We have then included in the fits the points from the correlation in the space directions up to $t=10$ only. For the time direction which is twice longer the effect is negligible for the maximum values $(t=14)$ of the time distance where the signal is still appreciable.

Figures 1 and 2 give the flavour dependence of the static decay constants for the two values of $R_{2}$. In Tables 1 and 2 we present our detailed numerical results for the energy shift, for $Z_{L}$ and for the parameters of our simulations including the matched values of $\pi$ and $\rho$ masses. The extrapolation of the results to $n_{f}=+3$ has been attempted with a linear and a quadratic fit with the results collected in table 3 . While the former varies with the number of negative flavours included in the fit, the latter is more stable against this variation and leads to values which are compatible within our errors. This applies also to the case of $R_{2}=0.6$ where we have explored the flavour dependence up to $n_{f}=-8$. In order to take into account the 
error arising from an imperfect matching of some simulations, we have extrapolated the values of $Z_{L}$ corrected by the factor $\left(m_{\rho}^{n_{f}=0} / m_{\rho}^{n_{f}}\right)^{3 / 2}$. The unquenched estimates at $R_{2}=0.5$ and $R_{2}=0.6$ can be further extrapolated in the quark mass (i.e. in the inverse of the Wilson hopping parameter) to the chiral limit where a physical values of $f_{B}^{\text {static }}$ can be obtained by using the experimental value of the $\rho$ mass as an input.

Our final results for the unquenched $f_{B}^{\text {static }}$ at a lattice spacing corresponding to $\beta=5.7$ of the quenched case, extrapolated to the chiral limit with $n_{f}=3$ and not corrected for the renormalization constant is:

$$
f_{B}^{\text {static }} / Z^{\text {Ren }}=Z_{L} \sqrt{\frac{2}{M_{B}}}\left(\frac{m_{\rho}^{\text {phys }}}{m_{\rho}^{\text {lattice }}}\right)^{3 / 2}=0.66(7) \mathrm{GeV}
$$

to be compared with the quenched result:

$$
f_{B}^{\text {static }} / Z^{\text {Ren }}=0.50(3) \mathrm{GeV}
$$

The unquenched estimate for the ratio $f_{B_{s}}^{\text {static }} / f_{B_{u}}^{\text {static }}$ cannot be obtained directly from the quark mass dependence of our results. Indeed, in our simulations we have identified the valence and the sea quark masses. This is appropriate for the light quark spectrum, while for the strange quark spectroscopy one should keep the two types of masses distinct. We defer such a refined study to a future work.

Including dynamical fermion loops increases the static decay constants, an effect already claimed by the MILC collaboration which uses dynamical staggered quarks [8].

The results discussed so far are for the bare quantities, not corrected for the appropriate renormalization constants. Their inclusion partially compensates the observed flavour dependence, but the effect can be estimated to be smaller than the increase of $Z_{L}$ due to the unquenching. Indeed by using the bare couplings in the one loop expression for $Z^{R e n}$, the percentage of the unquenching effects is decreased by a few units. By using an improved perturbation theory, i.e. a coupling constant in a Parisi [9] scheme, the effect is larger and the percentage decreases by about ten units. The extrapolated values of $Z^{\text {Ren }}$ in the Parisi coupling scheme are given in Table 3.

A sizeable flavour dependence survives the corrections of the renormalization constant, quantifies into a 10-15\% increase in the intermediate quark 
mass region that we have explored and becomes about $20 \%$ in the chiral limit.

\section{References}

[1] E. Eichten, B. Hill, Phys. Lett. B 232 (1989) 113.

[2] R. Petronzio, Nucl. Phys. B (Proc. Suppl.) 42 (1995) 942;

G. M. de Divitiis, R. Frezzotti, M. Guagnelli, M. Masetti, R. Petronzio, Nucl. Phys. B 455 (1995) 274;

G. M. de Divitiis, R. Frezzotti, M. Guagnelli, M. Masetti, R. Petronzio, Ph. Lett. B 367 (1996) 279.

[3] G. M. de Divitiis, R. Frezzotti, M. Masetti, R. Petronzio, "Pseudofermion observables for static heavy meson decay constants on the lattice", preprint ROM2F-96-10, submitted to Ph. Lett. B.

[4] G. Parisi, R. Petronzio, C. Rapuano, Phys. Lett. B 128 (1983) 418.

[5] G. M. de Divitiis, R. Frezzotti, M. Guagnelli, M. Masetti, R. Petronzio, Ph. Lett. B 353 (1995) 274.

[6] N. Cabibbo, E. Marinari, Ph. Lett. B 119 (1982) 387.

[7] M. Lüscher, Nucl. Phys. B 418 (1994) 637; B. Bunk, K. Jansen, B. Jegerlehner, M. Lüscher, H. Simma, R. Sommer, Nucl. Phys. B (Proc. Suppl.) 42 (1995) 49.

[8] C. Bernard et al., Lattice '95 proceedings; Nucl. Phys. B (Proc. Suppl.) (1996); preprint hep-lat 9509045.

[9] G. Parisi in the Proceedings of the High Energy Physics Conference, Madison, Wisconsin; Editors L. Durand and Lee G. Pondrom, AIP New York (1981) 1531 


\begin{tabular}{||c|c|c|c|c|c|c|c||}
\hline$n_{f}$ & $n_{\text {meas }}$ & $\beta$ & $\kappa$ & $\Delta E$ & $Z_{L}$ & $m_{\pi}$ & $m_{\rho}$ \\
\hline-8 & 6000 & 6.99 & 0.153 & $0.742(2)$ & $0.440(2)[7]$ & $0.563(5)$ & $0.712(10)$ \\
-6 & 5000 & 6.71 & 0.1548 & $0.751(3)$ & $0.466(2)[8]$ & $0.559(6)$ & $0.716(9)$ \\
-4 & 3000 & 6.4 & 0.157 & $0.770(3)$ & $0.515(4)[9]$ & $0.552(3)$ & $0.710(6)$ \\
-2 & 4400 & 6.06 & 0.1598 & $0.789(2)$ & $0.573(2)[7]$ & $0.566(4)$ & $0.714(8)$ \\
0 & 9200 & 5.7 & 0.163 & $0.811(1)$ & $0.648(4)[4]$ & $0.562(2)$ & $0.719(4)$ \\
\hline
\end{tabular}

Table 1: Results for $\Delta E$ and $Z_{L}$ at $R_{2}=0.6$ and the matched values of $m_{\pi}$ and $m_{\rho}$. The second column gives the number of measurements in each case. For $Z_{L}$ we separate the statistical error () from the one coming from the determination of $\Delta E[]$.

\begin{tabular}{||c|c|c|c|c|c|c|c||}
\hline$n_{f}$ & $n_{\text {meas }}$ & $\beta$ & $\kappa$ & $\Delta E$ & $Z_{L}$ & $m_{\pi}$ & $m_{\rho}$ \\
\hline-6 & 3600 & 6.785 & 0.156 & $0.733(2)$ & $0.440(2)[5]$ & $0.477(8)$ & $0.67(1)$ \\
-4 & 3400 & 6.463 & 0.158 & $0.744(2)$ & $0.465(7)[6]$ & $0.464(4)$ & $0.658(8)$ \\
-2 & 5000 & 6.1 & 0.161 & $0.762(3)$ & $0.520(10)[9]$ & $0.467(2)$ & $0.662(5)$ \\
0 & 6200 & 5.7 & 0.165 & $0.786(2)$ & $0.590(7)[7]$ & $0.457(3)$ & $0.659(8)$ \\
\hline
\end{tabular}

Table 2: The same quantities of table 1 for $R_{2}=0.5$. 


\begin{tabular}{|c|c|c|c|c|c|c|c|}
\hline \multirow{3}{*}{$\begin{array}{c}\text { \# of } \\
\text { points }\end{array}$} & \multicolumn{7}{|c|}{$R_{2}=0.5$} \\
\hline & \multicolumn{2}{|c|}{$Z_{L}\left(n_{f}=3\right)$} & \multicolumn{2}{|c|}{$Z^{\operatorname{Ren}}\left(n_{f}=3\right)$} & \multicolumn{2}{|c|}{$Z_{L} Z^{\operatorname{Ren}}\left(n_{f}=3\right)$} & $\frac{Z_{L} Z^{\operatorname{Ren}}\left(n_{f}=3\right)}{Z_{L} Z^{\operatorname{Ren}}\left(n_{f}=0\right)}$ \\
\hline & parabolic & linear & parabolic & linear & parabolic & linear & parabolic \\
\hline 2 & - & $0.702(38)$ & - & 0.662 & - & $0.473(29)$ & - \\
\hline 3 & $0.744(55)$ & $0.681(22)$ & 0.647 & 0.670 & $0.490(42)$ & $0.465(17)$ & - \\
\hline \multirow[t]{4}{*}{4} & $0.730(23)$ & $0.655(17)$ & 0.649 & 0.678 & $0.485(18)$ & $0.453(14)$ & $1.16(4)$ \\
\hline & \multicolumn{7}{|c|}{$R_{2}=0.6$} \\
\hline & \multicolumn{2}{|c|}{$Z_{L}\left(n_{f}=3\right)$} & \multicolumn{2}{|c|}{$Z^{\operatorname{Ren}}\left(n_{f}=3\right)$} & \multicolumn{2}{|c|}{$Z_{L} Z^{\operatorname{Ren}}\left(n_{f}=3\right)$} & $\frac{Z_{L} Z^{\operatorname{Ren}}\left(n_{f}=3\right)}{Z_{L} Z^{\operatorname{Ren}}\left(n_{f}=0\right)}$ \\
\hline & parabolic & linear & parabolic & linear & parabolic & linear & parabolic \\
\hline 2 & - & $0.742(25)$ & - & 0.668 & - & $0.503(20)$ & - \\
\hline 3 & $0.779(42)$ & $0.730(17)$ & 0.659 & 0.673 & $0.519(33)$ & $0.499(13)$ & - \\
\hline 4 & $0.760(19)$ & $0.730(13)$ & 0.659 & 0.678 & $0.506(15)$ & $0.504(11)$ & - \\
\hline 5 & $0.778(12)$ & $0.718(12)$ & 0.661 & 0.684 & $0.522(9)$ & $0.498(9)$ & $1.14(2)$ \\
\hline
\end{tabular}

Table 3: We give the results for the values of $Z_{L}$ extrapolated to $n_{f}=3$ at $R_{2}=0.5$ and $R_{2}=0.6$ as a function of the number of points used in the extrapolation. 


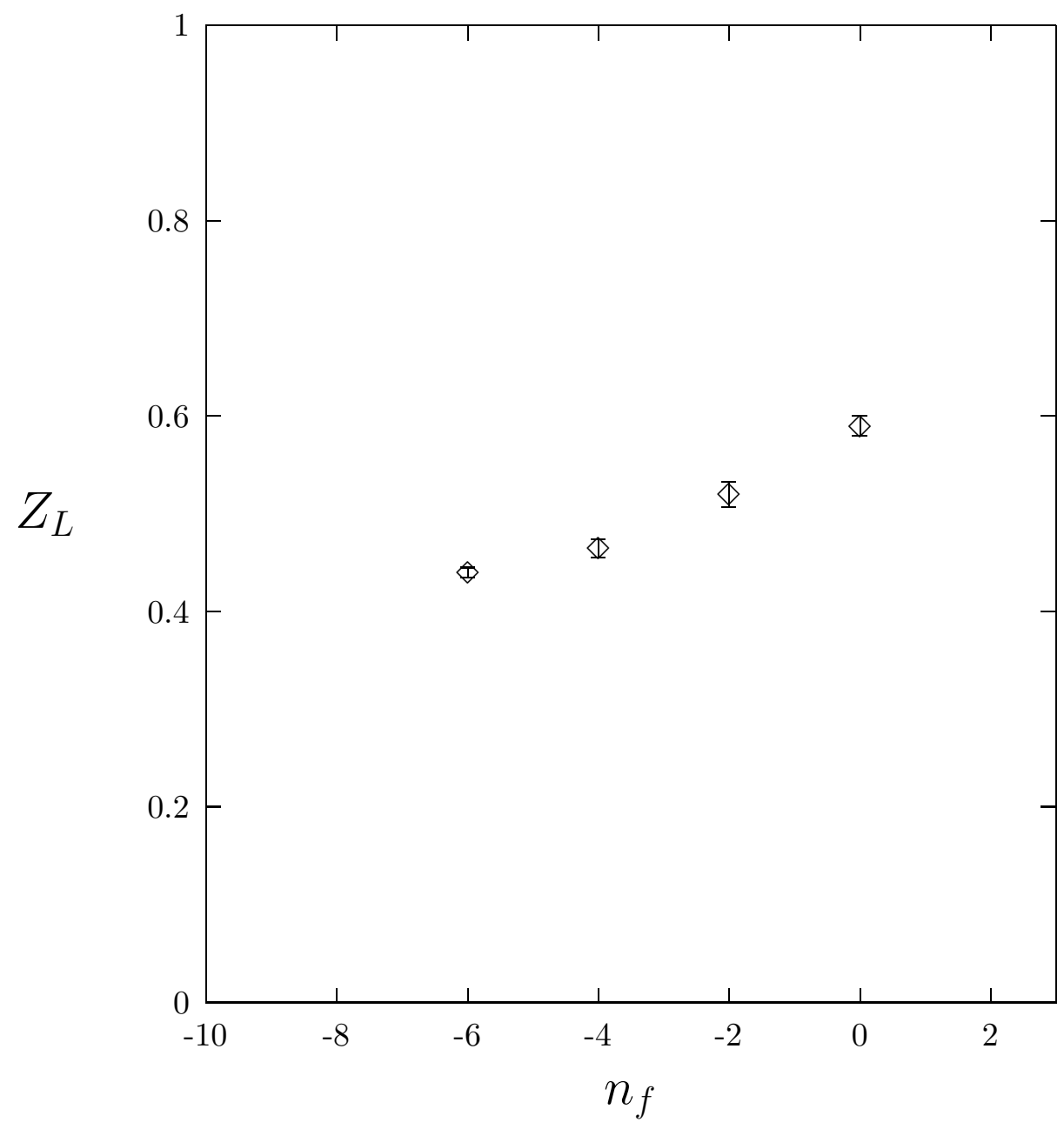

Figure 1: The values of $Z_{L}$ as a function of the flavour number for $R_{2}=0.5$. 


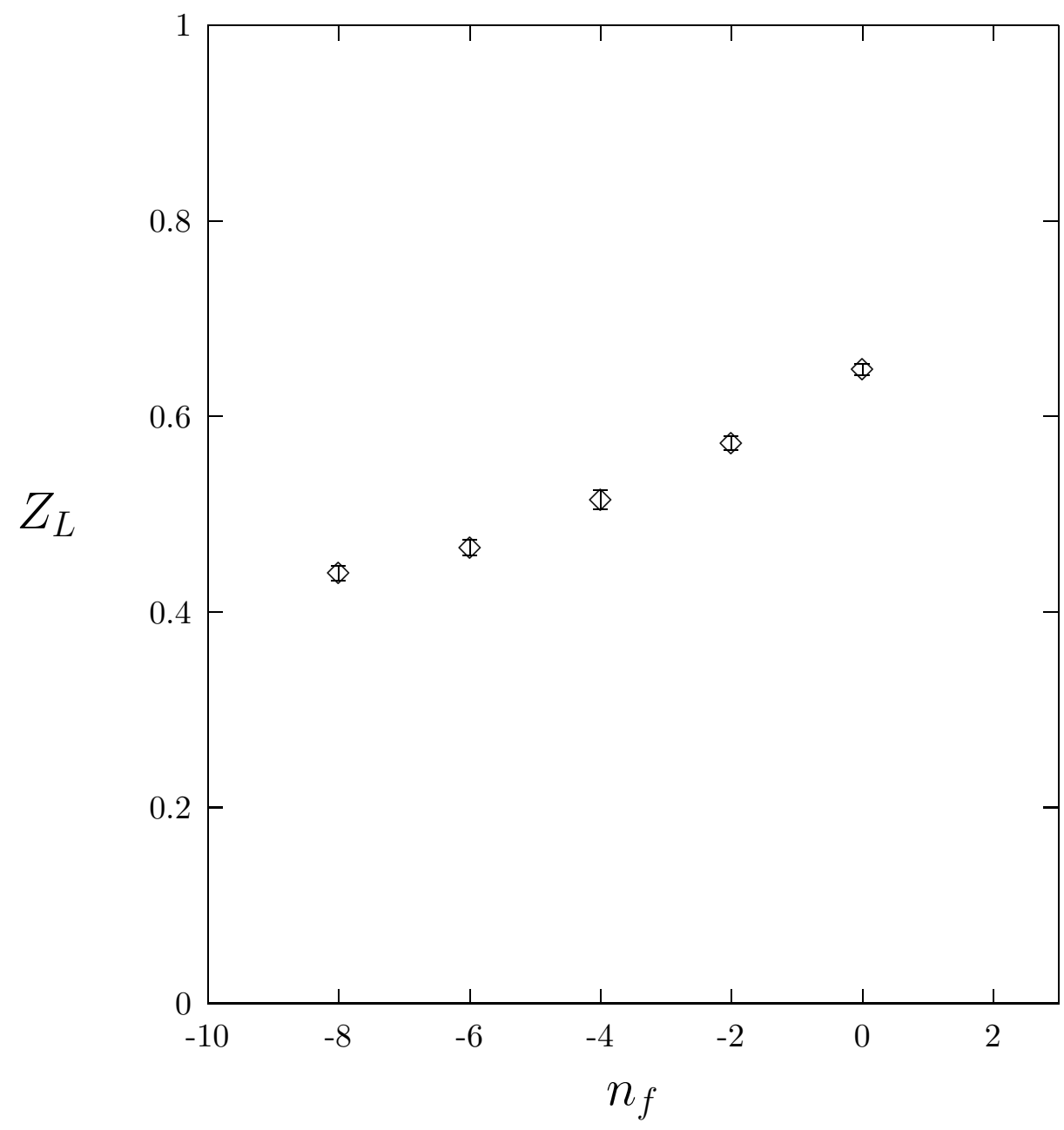

Figure 2: The values of $Z_{L}$ as a function of the flavour number for $R_{2}=0.6$. 